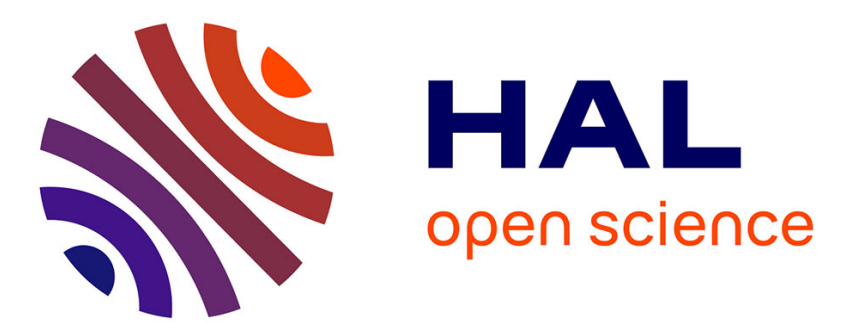

\title{
On the dipole, velocity and acceleration forms in high-order harmonic generation from a single atom or molecule
}

\author{
Jan Conrad Baggesen, Lars Bojer Madsen
}

\section{- To cite this version:}

Jan Conrad Baggesen, Lars Bojer Madsen. On the dipole, velocity and acceleration forms in highorder harmonic generation from a single atom or molecule. Journal of Physics B: Atomic, Molecular and Optical Physics, 2011, 44 (11), pp.115601. 10.1088/0953-4075/44/11/115601 . hal-00625578

\section{HAL Id: hal-00625578 \\ https://hal.science/hal-00625578}

Submitted on 22 Sep 2011

HAL is a multi-disciplinary open access archive for the deposit and dissemination of scientific research documents, whether they are published or not. The documents may come from teaching and research institutions in France or abroad, or from public or private research centers.
L'archive ouverte pluridisciplinaire HAL, est destinée au dépôt et à la diffusion de documents scientifiques de niveau recherche, publiés ou non, émanant des établissements d'enseignement et de recherche français ou étrangers, des laboratoires publics ou privés. 


\title{
On the dipole, velocity and acceleration forms in high-order harmonic generation from a single atom or molecule
}

\section{Jan Conrad Baggesen and Lars Bojer Madsen}

Lundbeck Foundation Theoretical Center for Quantum System Research, Department of Physics and Astronomy, Aarhus University, 8000 Aarhus C, Denmark

\begin{abstract}
We consider high-order harmonic generation from a single atom or molecule and show that the generated harmonic field is proportional to the dipole velocity. We derive this result by solving Maxwell's wave equation for an infinitely thin gas. Hence, the dipole velocity form is the one that relates directly to the harmonic field, and it should be used as a reference if performing calculations with the dipole or acceleration forms.
\end{abstract}

PACS numbers: $32.80 . \mathrm{Rm}$ 


\section{Introduction}

The full theory of high-order harmonic generation (HHG) is extremely complicated to evaluate. Such a theory should account for the build-up of the HHG field starting from zero photons, and accordingly it involves a quantum description of the emerging and propagating electromagnetic field. A simpler version of the above involves the propagation of Maxwell's equations for a classical electromagnetic field through a medium in combination with a quantum description of the polarization (see reference [1] for a review). Even this description is computationally demanding, in particular if the quantum mechanical input for the polarization is obtained by solving the timedependent Schrödinger equation (TDSE) [1] and even more so if the target gas consists of molecules [2,3], where lack of spherical symmetry impedes a reduction in the dimensionality of the problem. Accordingly, to simplify the description, most theory works, aiming at an evaluation of the HHG spectrum of specific atoms and molecules, are concerned with the description of the response of a single quantum system to the external driving field, and indeed this approach has over the years accounted for many important features of the experimental data (see, for example [4,5] and references therein).

A central question is then how the spectrum is related to the response from a single atom or molecule. Does it relate directly to the dipole $\left(D_{z}\right)$,

$$
S_{d i p}(\omega) \propto\left|\int_{-\infty}^{\infty} d t\left\langle\Psi(t)\left|D_{z}\right| \Psi(t)\right\rangle e^{i \omega t}\right|^{2},
$$

the dipole velocity $\left(\dot{D}_{z}\right)$

$$
S(\omega) \propto\left|\int_{-\infty}^{\infty} d t\left\langle\Psi(t)\left|\dot{D}_{z}\right| \Psi(t)\right\rangle e^{i \omega t}\right|^{2},
$$

or the dipole acceleration $\left(\ddot{D}_{z}\right)$

$$
S_{a c c}(\omega) \propto\left|\int_{-\infty}^{\infty} d t\left\langle\Psi(t)\left|\ddot{D}_{z}\right| \Psi(t)\right\rangle e^{i \omega t}\right|^{2},
$$

for a pump linearly polarized along $z$ and for a system described by $|\Psi(t)\rangle$ ? This question was addressed in reference [6], where the HHG spectrum was related to the norm squared of the Fourier transform of the dipole acceleration. Also references [7-9] related the HHG spectrum to the dipole acceleration. In a more recent work [10], however, a quantum-electrodynamical approach was taken and results corresponding to the ones of reference [6] were obtained except that the spectrum was shown to relate naturally to the dipole velocity. Currently, however, there is no consensus in the literature of what quantity to calculate in order to predict the harmonic spectrum generated by a single atom or molecule. For example in references [11-13] the harmonic signal is calculated as the norm square of the Fourier transform of the time-dependent dipole (equation (1)), while in references [14-18] the spectrum is calculated as the norm squared of the Fourier transform of the time-dependent dipole acceleration (equation (3)). But in fact, as discussed here, the correct approach in the single-system limit is 
to calculate the spectra using the dipole velocity form as a reference, and refer back to the result using that form by accounting for appropriate factors of $\omega$ (see equations (11)-(12) below), if the dipole or acceleration forms were used. In the following we shall refer to the quantities $S_{d i p}$ and $S_{a c c}$ from equations (1) and (3) as the result of the dipole and accelerations forms. The quantity $S$, based on the velocity form, will be shown to give the harmonic signal.

The correct choice of reference form is important as illustrated by noting that if the spectrum calculated is extended up to the 100th harmonic order, as is not uncommon, the difference in the scaling with the frequency $\omega$ of the harmonic between dipole and dipole acceleration forms can be as much as 8 orders of magnitude. Further, as some of the interesting features in the harmonic spectra are quite shallow dips [19-21], the scaling may push these dips to different positions and hence change the interpretation. Finally, the establishment of a fixed reference form is important for quantitative comparisons of theoretical calculations and with experiments. In the case of macroscopic propagation such comparison is complicated by uncertainties in plasma frequencies, ionization rates and free-electron densities. Note in passing that the solution of the TDSE may be performed in any gauge and gauge invariant results will be obtained as long as appropriate unitary gauge transformation factors are accounted for $[22,23]$.

\section{Theory and discussion}

In our analytical approach, we consider an incoming light pulse $E_{i n}(t-x / c)$ propagating in the $x$ direction and linearly polarized along the $z$ direction. We place the atom or molecule in the origin and the fundamental field will induce a dipole in the quantum system and harmonics will be generated as described by an additional field $E_{g e n}(t-x / c)$. The resulting total electric field is denoted by $E(t-x / c)$. At a fixed observation point after the atom or molecule, the spectral distribution function $S(\omega)$ is given by (see, for example,reference [24])

$$
S(\omega)=|E(\omega)|^{2}=\frac{1}{4 \pi^{2}}\left|\int_{-\infty}^{\infty} E(t) e^{i \omega t} d t\right|^{2} .
$$

Hence, with the electric field at hand, the spectrum can easily be evaluated. To determine the electromagnetic field we consider Maxwell's wave equation in the propagation direction with a particular simple choice of the source polarization term

$$
\left(\frac{\partial^{2}}{\partial x^{2}}-\frac{1}{c^{2}} \frac{\partial^{2}}{\partial t^{2}}\right) E(x, t)=\frac{1}{\epsilon_{0} c^{2}} \frac{\partial^{2}}{\partial t^{2}}\left\langle D_{z}(t)\right\rangle \delta(x),
$$

where, for the system in state $|\Psi(t)\rangle,\left\langle D_{z}(t)\right\rangle=\left\langle\Psi(t)\left|D_{z}\right| \Psi(t)\right\rangle$ is the expectation value of the $z$ component of the atomic or molecular dipole, i.e. the $z$ component of $\vec{D}=e\left(\sum_{i} Z_{i} \vec{R}_{i}-\sum_{j} \vec{r}_{j}\right)$. The latter is assumed to be calculated in a quantum mechanical model, i.e., from a numerical solution of the TDSE or from the Lewenstein model [25]. As may be readily seen by insertion, the solution to equation (5) reads

$$
E(x, t)=E_{\text {in }}(t-x / c)
$$




$$
-\frac{1}{2 \epsilon_{0} c}\left[\theta(x)\left\langle\dot{D}_{z}(t-x / c)\right\rangle+\theta(-x)\left\langle\dot{D}_{z}(t+x / c)\right\rangle\right]
$$

where $\theta( \pm x)$ is the Heaviside step function with $\theta(x)=1$ for $x \geq 0$ and zero elsewhere, and $\left\langle\dot{D}_{z}(t \mp x / c)\right\rangle$ denotes the dipole velocity. In the second line of equation (6), we see that after the encounter with the single atom or molecule, two propagating waves are generated. One traveling in the propagation direction of the incoming pulse, $\left\langle\dot{D}_{z}(t-x / c)\right\rangle$, and one traveling in the opposite direction $\left\langle\dot{D}_{z}(t+x / c)\right\rangle$. The Heaviside functions ensure that the generated light propagates from the source located at $x=0$.

We imagine that the spectrum is measured at a fixed observation point in the forward propagation direction. After having passed the single atom or molecule, the solution of Maxwell's wave equation follows from equation (6)

$$
E(x, t)=E_{\text {in }}(t-x / c)-\frac{1}{2 \epsilon_{0} c}\left\langle\dot{D}_{z}(t-x / c)\right\rangle, \quad x>0 .
$$

The spectrum of the electric pulse can now be determined from equation (4), and we obtain at the fixed observation point

$$
S(\omega)=\left|E_{\text {in }}(\omega)\right|^{2}+\left|E_{\text {gen }}(\omega)\right|^{2}+2 \operatorname{Re}\left(E_{\text {in }}(\omega)^{\star} E_{\text {gen }}(\omega)\right),
$$

where $E_{\text {in }}(\omega)=1 /(2 \pi) \int_{-\infty}^{\infty} E_{i n}(t) e^{i \omega t} d t$ is the Fourier transform of the incoming driving pulse and where

$$
E_{g e n}(\omega)=-\frac{1}{2 \epsilon_{0} c} \frac{1}{2 \pi} \int_{-\infty}^{\infty}\left\langle\dot{D}_{z}(t)\right\rangle e^{i \omega t}=-\frac{1}{2 \epsilon_{0} c} \dot{D}_{z}(\omega)
$$

is the generated electric field in the frequency domain, and where we have introduced the notation $\dot{D}_{z}(\omega)=1 /(2 \pi) \int_{-\infty}^{\infty}\left\langle\dot{D}_{z}(t)\right\rangle e^{i \omega t}$. Equation (9) expresses clearly the generated field in terms of the dipole velocity. This result can also be derived from equation (58) of [26] including phasematching effects, and by solving the Maxwell equation in the slowly-varying amplitude approximation.

In typical HHG experiments, the incoming pump laser pulse does not have any spectral density at the relatively high frequencies of interest in HHG and equation (8) reduces to

$$
S(\omega)=\left|E_{\text {gen }}(\omega)\right|^{2}=\frac{1}{4 \epsilon_{0}^{2} c^{2}}\left|\dot{D}_{z}(\omega)\right|^{2},
$$

and this result shows that equation (2) is the correct one to use. In case it is important to include relaxation processes, for example related to the damping of the induced dipole by radiative decay, the expectation value is most readily calculated by $\left\langle\dot{D}_{z}(t)\right\rangle=\operatorname{tr}\left(\rho(t) \dot{D}_{z}\right)$, where $\rho(t)$ is the density matrix of the system at time $t$. In most TDSE calculations, however, only coherent evolution and a single initial state is considered and the expectation value is determined from $\left\langle\dot{D}_{z}(t)\right\rangle=\left\langle\Psi(t)\left|\dot{D}_{z}\right| \Psi(t)\right\rangle$, where $|\Psi(t)\rangle$ is the time-evolved initial state. In the case of the single-active-electron approximation, which is widely used in strong-field physics, the above dipole simplifies to $\dot{D}_{z}=-e \dot{z}$, where the notation $\dot{z}$ stresses that it is the kinematical momentum $m_{e} \dot{z}$ that is involved in the generation of harmonics and not the canonical momentum. The 


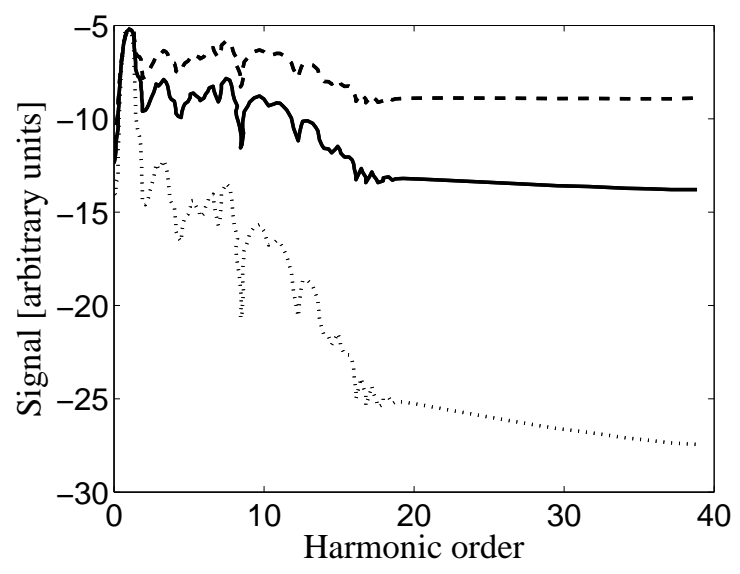

Figure 1. High-order harmonic spectrum from hydrogen as a function of harmonic order for a 3 cycle pulse at a wavelength of $800 \mathrm{~nm}$ and an intensity of $3 \times 10^{13} \mathrm{Wcm}^{-2}$. The full curve shows $\log _{10}\left(\mid\left(\left.\dot{D}_{z}(\omega)\right|^{2}\right)\right.$, which is the signal up to trivial constants (see equation (10)) based on the velocity dipole form and is taken from reference [23]. The dashed curve shows $\log _{10}\left(\omega^{2} \mid\left(\left.\dot{D}_{z}(\omega)\right|^{2}\right)\right.$ which corresponds to the acceleration form, equation (3) and finally the dotted curve shows $\log _{10}\left(\mid\left(\left.\dot{D}_{z}(\omega)\right|^{2} / \omega^{2}\right)\right.$, which corresponds to the dipole form, equation (1). The curves have been scaled to the first peak in the spectrum of the full curve.

formulation is accordingly suitable also in the velocity gauge where the kinematical and canonical momenta differ by the vector potential, $m_{e} \dot{z}=p_{z}+e A$.

We now consider the relation of the spectrum to the formulation with the dipole and the dipole acceleration forms. If we assume that at large negative and positive times, that is long before and after the pulse, $D_{z}(t)=0$ and $\dot{D}_{z}(t)=0$ (see references $[9,23,27]$ for more general cases) partial integration gives

$$
\dot{D}_{z}(\omega)=-i \omega D_{z}(\omega)
$$

and

$$
\dot{D}_{z}(\omega)=\frac{i}{\omega} \ddot{D}(\omega)
$$

and the corresponding spectra are obtained from equation (10), as

$$
\begin{aligned}
& S(\omega)=\frac{\omega^{2}}{4 \epsilon_{0}^{2} c^{2}}\left|D_{z}(\omega)\right|^{2}, \\
& S(\omega)=\frac{1}{4 \epsilon_{0}^{2} c^{2}} \frac{1}{\omega^{2}}\left|\ddot{D}_{z}(\omega)\right|^{2} .
\end{aligned}
$$

Of particular interest here is the scaling with $\omega$. Clearly if the dipole acceleration as in equation (3) were taken as the fundamental form, one would obtain a different scaling with harmonic frequency $\omega$ in equations (10), (13), and (14). In that case the dipole velocity would be multiplied by $\omega^{2}$ and the dipole form by $\omega^{4}$, which was the underlying assumption in early works [6-9]. In other words, the way the spectra decrease as a function of $\omega$ depends on which form is taken as the fundamental reference from which to derive the spectra based on the other forms. 


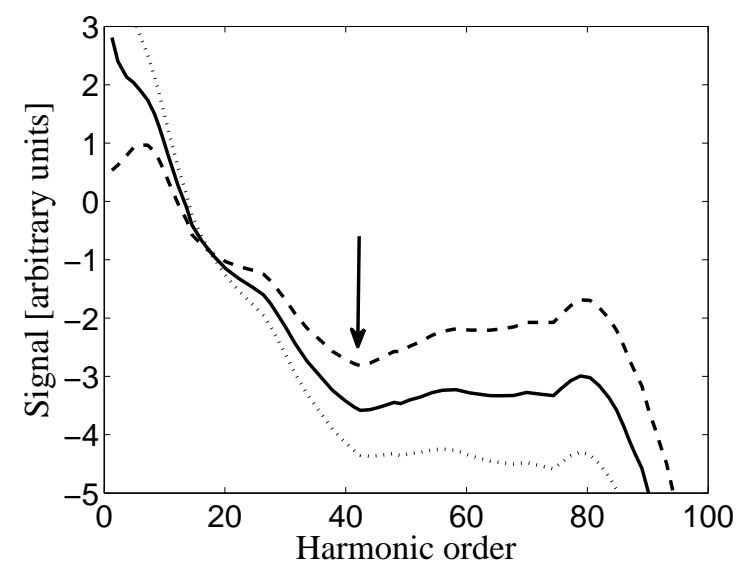

Figure 2. Smoothed high-order harmonic spectrum for the hydrogen molecular ion for a 10 cycle pulse at $780 \mathrm{~nm}$ and peak intensity of $5 \times 10^{14} \mathrm{Wcm}^{-2}$. The dashed curve is the acceleration form result $\log _{10}\left(\mid\left(\left.\ddot{D}_{z}(\omega)\right|^{2}\right)\right.$ taken from [19]. The full curve is the result with the velocity dipole form, $\log _{10}\left(\mid\left(\left.\dot{D}_{z}(\omega)\right|^{2} / \omega^{2}\right)\right.$, and the dotted curve is the result of the dipole form, $\log _{10}\left(\mid\left(\left.D_{z}(\omega)\right|^{2} / \omega^{4}\right)\right.$. The arrow indicates the position of the minimum at the $43 \mathrm{rd}$ harmonic as predicted by the acceleration form.

Figure 1 illustrates this point. In the figure, the HHG spectrum for hydrogen is considered as a function of harmonic order for a 3 cycle pulse with a sine-squared envelope for the vector potential and of $800 \mathrm{~nm}$ and a peak intensity of $3 \times 10^{13} \mathrm{Wcm}^{-2}$. The full curve, taken from reference [23], shows $\log _{10}\left(\mid\left(\left.\dot{D}_{z}(\omega)\right|^{2}\right)\right.$, which is the signal up to trivial constants (see equation (10)) based on the velocity dipole form, and as shown above this is the form that relates directly to the field generated from a single atom or molecule. If erroneously the acceleration form of equation (3) were taken as the fundamental reference form, equation (12) would predict the result $\log _{10}\left(\omega^{2} \mid\left(\left.\dot{D}_{z}(\omega)\right|^{2}\right)\right.$ shown by the dashed curve, and finally if - again by error - the dipole form of equation (1) were taken as the reference, equation (11) would predict the result $\log _{10}\left(\mid\left(\left.\dot{D}_{z}(\omega)\right|^{2} / \omega^{2}\right)\right.$ shown by the dotted curve in figure 1 . As seen from the figure the dashed and the dotted curves generally differ from the correct full curve and in particular they, respectively, over and under estimate the signal at high order. Another consequence of the different scaling of the three forms is a change in minima positions and structures in the HHG spectra from molecules. Such minima may result from a destructive interference when the electron after its excursion in the continuum recombines under the influence of different atomic centers, and the position of minima reflects the atomic positions at the instant of recombination as predicted [19] and observed [20,21]. In figure 2 we illustrate the behaviour of the minimum in different forms. While the minimum is present in the case of the acceleration (dashed curve) and the velocity (full curve) form, the minimum has almost disappeared in the case of the dipole length form (dotted curve).

It is important to note that our aim here is to describe the single system response and to set a standard for the choice of reference form when presenting theoretical HHG spectra. As discussed in the introduction, many calculations of high-order 
harmonic spectra are indeed carried out for single systems and the results of many HHG experiments are interpreted in terms of the single system response. This approach is clearly an approximation since the full description of HHG requires inclusion of propagation through the medium, phase-matching effects, and absorption [1]. Other works have focused on these aspects and propagation effects may be very important, depending on the experimental conditions. For example substantial efforts have been put into the calculation of macroscopic effects on the HHG spectrum [5, 26, 28, 29], the influence on the generation of attosecond pulses [30,31] and also to investigate the effects of propagation on more delicate structures such as the Cooper minimum in argon [32]. In view these works it is clear that no spectrum obtained by considering a single system can capture the full macroscopic response.

\section{Conclusion}

In conclusion, we have derived the harmonic field generated by the response of a single atom or molecule to the fundamental pump field. We did so by solving Maxwell's wave equation analytically for an atom or molecule placed at the origin. The result shows that the spectrum of HHG directly relates to the Fourier transform of the expectation value of the time-dependent dipole velocity, in agreement with recent results derived using quantum electrodynamics [10]. While the source term entering the right-hand side of Maxwell's wave equation (5) is the dipole acceleration, the term entering the resulting electric field is the dipole velocity. The significance of using the correct form for a fixed reference was illustrated by considering HHG from hydrogen, and the hydrogen molecular ion and it was illustrated how using the wrong form could artificially enhance or weaken the high-energy part of the spectrum. Other characteristics of the spectra are also modified. In particular the minima in the spectrum shifts. Such modifications may be of importance when using HHG as a spectroscopic tool for the retrieval of atomic positions or orbitals [33]. The present work shows that the velocity form should be used as the standard reference, a fact that will ease the comparison between different theoretical calculations in the future.

\section{Acknowledgments}

We thank Christian Bruun Madsen for useful discussions. The present work was supported by the Danish National Research Council (Grant number 10-85430).

\section{References}

[1] Mette B Gaarde, Jennifer L Tate, and Kenneth J Schafer. Macroscopic aspects of attosecond pulse generation. Journal of Physics B: Atomic, Molecular and Optical Physics, 41(13):132001, 2008.

[2] E. Lorin, S. Chelkowski, and A. Bandrauk. A numerical maxwell-schroedinger model for intense 
laser-matter interaction and propagation. Computer Physics Communications, 177(12):908 932, 2007.

[3] E Lorin, S Chelkowski, and A D Bandrauk. Attosecond pulse generation from aligned moleculesdynamics and propagation in h 2 +. New Journal of Physics, 10(2):025033, 2008.

[4] Cheng Jin, Anh-Thu Le, and C. D. Lin. Retrieval of target photorecombination cross sections from high-order harmonics generated in a macroscopic medium. Phys. Rev. A, 79(5):053413, May 2009.

[5] Cheng Jin, Anh-Thu Le, and C. D. Lin. Medium propagation effects in high-order harmonic generation of ar and $n_{2}$. Phys. Rev. A, 83(2):023411, Feb 2011.

[6] Bala Sundaram and Peter W. Milonni. High-order harmonic generation: Simplified model and relevance of single-atom theories to experiment. Phys. Rev. A, 41(11):6571-6573, Jun 1990.

[7] J. H. Eberly and M. V. Fedorov. Spectrum of light scattered coherently or incoherently by a collection of atoms. Phys. Rev. A, 45(7):4706-4712, Apr 1992.

[8] D. G. Lappas, M. V. Fedorov, and J. H. Eberly. Spectrum of light scattered by a strongly driven atom. Phys. Rev. A, 47(2):1327-1335, Feb 1993.

[9] K. Burnett, V. C. Reed, J. Cooper, and P. L. Knight. Calculation of the background emitted during high-harmonic generation. Phys. Rev. A, 45(5):3347-3349, Mar 1992.

[10] D. J. Diestler. Harmonic generation: Quantum-electrodynamical theory of the harmonic photonnumber spectrum. Phys. Rev. A, 78(3):033814, Sep 2008.

[11] André D. Bandrauk, Szczepan Chelkowski, Shinnosuke Kawai, and Huizhong Lu. Effect of nuclear motion on molecular high-order harmonics and on generation of attosecond pulses in intense laser pulses. Phys. Rev. Lett., 101(15):153901, Oct 2008.

[12] Jing Zhao and Zengxiu Zhao. Probing $h_{2}^{+}$vibrational motions with high-order harmonic generation. Phys. Rev. A, 78(5):053414, Nov 2008.

[13] P. B. Corkum. Plasma perspective on strong field multiphoton ionization. Phys. Rev. Lett., 71(13):1994-1997, Sep 1993.

[14] J. Tate, T. Auguste, H. G. Muller, P. Salières, P. Agostini, and L. F. DiMauro. Scaling of wavepacket dynamics in an intense midinfrared field. Phys. Rev. Lett., 98(1):013901, Jan 2007.

[15] Misha Yu. Ivanov, Thomas Brabec, and Neal Burnett. Coulomb corrections and polarization effects in high-intensity high-harmonic emission. Phys. Rev. A, 54(1):742-745, Jul 1996.

[16] M. Lein, N. Hay, R. Velotta, J. P. Marangos, and P. L. Knight. Role of the intramolecular phase in high-harmonic generation. Phys. Rev. Lett., 88(18):183903, Apr 2002.

[17] J. Levesque, D. Zeidler, J. P. Marangos, P. B. Corkum, and D. M. Villeneuve. High harmonic generation and the role of atomic orbital wave functions. Phys. Rev. Lett., 98(18):183903, May 2007.

[18] Emmanuel Penka Fowe and Andre D. Bandrauk. Nonlinear time-dependent density-functionaltheory study of ionization and harmonic generation in $\mathrm{Co}_{2}$ by ultrashort intense laser pulses: Orientational effects. Phys. Rev. A, 81(2):023411, Feb 2010.

[19] M. Lein, N. Hay, R. Velotta, J. P. Marangos, and P. L. Knight. Interference effects in high-order harmonic generation with molecules. Phys. Rev. A, 66(2):023805, Aug 2002.

[20] Tsuneto Kanai, Shinichirou Minemoto, and Hirofumi Sakai. Quantum interference during highorder harmonic generation from aligned molecules. Nature, 435(7041):470-474, May 2005.

[21] C. Vozzi, F. Calegari, E. Benedetti, J.-P. Caumes, G. Sansone, S. Stagira, M. Nisoli, R. Torres, E. Heesel, N. Kajumba, J. P. Marangos, C. Altucci, and R. Velotta. Controlling two-center interference in molecular high harmonic generation. Phys. Rev. Lett., 95(15):153902, Oct 2005.

[22] Lars Bojer Madsen. Gauge invariance in the interaction between atoms and few-cycle laser pulses. Phys. Rev. A, 65(5):053417, May 2002.

[23] Yong-Chang Han and Lars Bojer Madsen. Comparison between length and velocity gauges in quantum simulations of high-order harmonic generation. Phys. Rev. A, 81(6):063430, Jun 2010.

[24] Rodney Loudon. The quantum theory of light. Clarendon Press, Second Edition, Oxford, 1983.

[25] M. Lewenstein, Ph. Balcou, M. Yu. Ivanov, Anne L'Huillier, and P. B. Corkum. Theory of high- 
harmonic generation by low-frequency laser fields. Phys. Rev. A, 49(3):2117-2132, Mar 1994.

[26] B. W. Shore and K. C. Kulander. Generation of optical harmonics by intense pulses of laser radiation - i. propagation effects. Journal of Modern Optics, 36(7):857-875, July 1989.

[27] A. D. Bandrauk, S. Chelkowski, D. J. Diestler, J. Manz, and K.-J. Yuan. Quantum simulation of high-order harmonic spectra of the hydrogen atom. Phys. Rev. A, 79(2):023403, Feb 2009.

[28] A. L'Huillier, L.-A. Lompre, G. Mainfray, and C. Manus. High-order harmonic generation in rare gases. in Atoms in intense laser fields, ed. M. Gavrila (Academic Press, 1992).

[29] E. Constant, D. Garzella, P. Breger, E. Mevel, Ch. Dorrer, C. Le Blanc, F. Salin, and P. Agostini. Optimizing high harmonic generation in absorbing gases: Model and experiment. Phys. Rev. Lett., 82(8):1668-1671, Feb 1999.

[30] A. S. Sandhu, E. Gagnon, A. Paul, I. Thomann, A. Lytle, T. Keep, M. M. Murnane, H. C. Kapteyn, and I. P. Christov. Generation of sub-optical-cycle, carrier-envelope-phase-insensitive, extremeuv pulses via nonlinear stabilization in a waveguide. Phys. Rev. A, 74(12):061803(R), Dec 2006.

[31] T Tuchon, C P Hauri, K Varj, E Mansten, M Swoboda, R Lopez-Martens, and A L'Huillier. Macroscopic effects in attosecond pulse generation. New Journal of Physics, 10(2):025027, 2008.

[32] J. P. Farrell, L. S. Spector, B. K. McFarland, P. H. Bucksbaum, M. Guhr, M. B. Gaarde, and K. J. Schafer. Influence of phase matching on the Cooper minimum in Ar high-order harmonic spectra. Phys. Rev. A, 83(2):023420, Feb 2011.

[33] J. Itatani, J. Levesque, D. Zeidler, Hiromichi Niikura, H. Pepin, J. C. Kieffer, P. B. Corkum, and D. M. Villeneuve. Tomographic imaging of molecular orbitals. Nature, 432(7019):867-871, Dec 2004 . 